OPEN ACCESS

Edited by:

Rhonda Swickert,

College of Charleston, United States

Reviewed by:

Snezana Smederevac,

University of Novi Sad, Serbia

Jared Ruchensky,

Sam Houston State University,

United States

*Correspondence:

Anders Carlander

anders.carlander@gu.se

Specialty section:

This article was submitted to

Personality and Social Psychology,

a section of the journal

Frontiers in Psychology

Received: 17 June 2020

Accepted: 27 October 2020

Published: 13 November 2020

Citation:

Carlander A and Johansson L-O (2020) Should Trust Be Stressed? General Trust and Proactive Coping

as Buffers to Perceived Stress.

Front. Psychol. 11:554962.

doi: 10.3389/fpsyg.2020.554962

\section{Should Trust Be Stressed? General Trust and Proactive Coping as Buffers to Perceived Stress}

\author{
Anders Carlander ${ }^{1,2 *}$ and Lars-Olof Johansson ${ }^{3}$ \\ ${ }^{1}$ SOM Institute, University of Gothenburg, Gothenburg, Sweden, ${ }^{2}$ Wallenberg Centre for Molecular and Translational \\ Medicine, University of Gothenburg, Gothenburg, Sweden, ${ }^{3}$ Department of Psychology, University of Gothenburg, \\ Gothenburg, Sweden
}

Stress is becoming an increasingly important public health concern. Assuming that individual levels of trust and coping can buffer psychological stress, we explore validated measures of general trust [General Trust Scale (GTS)], proactive coping [Proactive Coping Inventory $(\mathrm{PCl})$ ], jointly with personality [Honesty-Humility, Emotionality, Extraversion, Agreeableness, Conscientiousness, and Openness to experience (HEXACO)], and intolerance of uncertainty (IUS), as predictors of perceived stress [Perceived Stress Scale (PSS)]. Data were collected from Qualtrics research panels using quota sampling to obtain two representative American community samples. The assumed alleviating effects of GTS and $\mathrm{PCI}$ on PSS remained but were attenuated when modeled jointly with HEXACO, IUS, and socio-economic background variables [socioeconomic status (SES)] in hierarchical regressions. In Study 1 ( $N=1,213)$, SES explained 19\% and HEXACO explained $29 \%$ of the variance in PSS. Introducing IUS and GTS added significant but small portions of explained variance. In Study 2 ( $N=1,090$ ), after controlling for SES which explained 18\% of the variance, IUS explained an additional 18\% of the variance in PSS. Adding GTS to the model showed modest contributions whereas PCl added 9\% of explained variance in the final hierarchical step. The findings highlight that GTS and PCI remain important variables even after controlling well-known factors such as personality and ability to tolerate uncertainty. However, given the weak effects of GTS, to consider trust as a remedy for stress may be of limited use in clinical practice since it could potentially be explained largely as a proxy for a beneficial combination of personality, coping, and socioeconomic background.

Keywords: stress, personality, intolerance of uncertainty, coping, trust

\section{INTRODUCTION}

Psychological stress is an increasingly important issue (APA, 2020) with potentially important implications for people's health (Schneiderman et al., 2005; Folkman and Nathan, 2011), and cognitive function (Lupien et al., 2009; Pechtel and Pizzagalli, 2011) and may be seen as a risk factor for psychopathology (Charles et al., 2013; Salim, 2014). In understanding what may drive or alleviate psychological stress, individual factors such as personality and coping strategies 
are important (Carver and Connor-Smith, 2010). Recently, the health benefits of trust have attracted attention not only in psychology but also in the fields of medicine and public health (Kawachi et al., 2008; Giordano and Lindström, 2016; Giordano et al., 2019). As health-promoting factors, the efficacy and relevance of related contextual effects and similar constructs to general trust also need to be evaluated (Shiell et al., 2020).

Consistent with the stress-buffering hypothesis of social support (Cohen and Wills, 1985), we posit that trust may function as a coping mechanism when people face stressors. Measures of trust and coping have however rarely been modeled together with more dispositional factors such as personality and intolerance for uncertainty. In line with Shiell et al. (2020), we recognize a need to investigate and disentangle the alleged stress-buffering effects of trust. We pursue this by testing how measures of general trust and proactive coping relate to perceived stress over and above what measures of personality and intolerance of uncertainty (IU) explain.

Psychological stress, commonly thought of as an interplay between cognitive and biological processes (Schneiderman et al., 2005), may be seen as an outcome of appraising threatening events that can result in stress reactions if and when coping resources prove to be inadequate (Lazarus and Folkman, 1984). Stress responses tend to vary with personality traits (Penley and Tomaka, 2002), often in conjunction with self-efficacy (Ebstrup et al., 2011). Psychological stress can be assessed using the Perceived Stress Scale (PSS), which measures the respondent's subjective stress level within a certain timeframe (Cohen et al., 1983).

Stress and negative emotions are furthermore shown to be positively associated with the Neuroticism trait and negatively related to the Extraversion, Agreeableness, Conscientiousness, and Openness traits from the Five Factor Model (FFM) of personality (Costa and McCrae, 1992). Moreover, previous research shows that different psychopathological conditions, including depression, anxiety disorders, phobias, and substance, use relate to higher Neuroticism and lower Extraversion and Conscientiousness (Kotov et al., 2010). It is generally believed that especially the Neuroticism trait will influence how individuals appraise threats and stressors, something which in turn may increase the risk of detrimental responses (Carver and ConnorSmith, 2010; Ebstrup et al., 2011). In addition, while Extraversion is related to more engaged coping strategies such as problemsolving and use of social support, Neuroticism is associated with disengaged coping strategies such as denial and substance use (Carver and Connor-Smith, 2010; Coulston et al., 2013). Given this, we believe that certain combinations of personality traits may influence and explain both how people appraise stressful events and how they engage their available coping resources.

Similar to the more common FFM (Costa and McCrae, 1992), the HEXACO personality inventory (which is used in the present research) is a trait model consisting of the six personality factors, Honesty-Humility (H), Emotionality (E), Extraversion (X), Agreeableness (A), Conscientiousness (C), and Openness (O) to experience (Lee and Ashton, 2004). A benefit of HEXACO is that the Honesty-Humility factor can be seen as the inverse to the shared variance of the dark triad of psychopathy, Machiavellianism, and narcissism (Lee and Ashton, 2005, 2014), which means that it functions well as a theoretically coherent and empirically independent complement to the more common FFM model. The measures of Honesty-Humility and Agreeableness are sometimes referred to as prosocial traits that may be positively linked to generosity and reciprocity (Zhao et al., 2016). Compared with the FFM model the additional Honesty-Humility factor offers some insights, particularly when studying socially relevant behaviors and attitudes.

Due to the necessity of anticipating future events, uncertainty requires an increased cognitive load. Previous research identifies individual differences in how well people tolerate uncertainty and ambiguity (Birrell et al., 2011). Originally developed by Freeston et al. (1994), IU was proposed as an important mechanism and antecedent to worry, seen as a trans-diagnostic construct, distinct from personality (McEvoy and Mahoney, 2012), and believed to be linked to several common psychopathologies including anxiety and mood disorders (Gentes and Ruscio, 2011; Carleton, 2016). Generally, IU deals with subjective notions of the unpredictability of negative events that can incur cognitive (aversion), emotional (anger, discomfort), and behavioral (avoidance, paralysis) responses, linked to both internalizing and externalizing psychopathology. IU can be assessed by the Intolerance of Uncertainty Scale (IUS, Freeston et al., 1994), which we use as a predictor in the present research. Higher scores on IUS is in general related to mood and anxiety disorders (Carleton et al., 2012), and the underlying factors of IU have been suggested to be linked to different behavioral outcomes in the face of uncertainty (see Birrell et al., 2011 for a review). The IUS and PSS measures have been reported to be positively related (Crum et al., 2013), something which may be explained by research showing that subjective uncertainty estimates induce subjective and physiological stress responses (De Berker et al., 2016). This implies that a higher dispositional sensitivity to uncertainty may lead to higher stress levels.

In relation to uncertainty, the concept of trust is often defined as a belief or intention to accept vulnerability based on a positive expectation regarding the intentions and behaviors of other people (Van Lange, 2015). Although trust has been researched in many scientific domains, the most common approach is to distinguish between trustworthiness (judgment of someone's ability, benevolence, integrity, etc.), trust propensity (the dispositional tendency to depend on others), and trust (the intention and behavior of entrusting someone; see Colquitt et al., 2007 for a meta-analysis). Furthermore, from a psychological perspective reasons for trusting include individual differences in disposition and personality, intergroup processes, and cognitive expectations of outcomes (Evans and Krueger, 2009). In other words, intention to trust may depend on a natural inclination to trust if there is a group or social norm encouraging trust and a beneficial outcome associated with trusting someone. The idea that trust can at least partly be seen as a dispositional characteristic (Rotter, 1971) or a personality trait (Costa and McCrae, 1992) is not new. Trust has largely been assumed to be an adaptive result of genes, social upbringing, 
and successful or aversive experiences (Glanville and Paxton, 2007; Van Lange, 2015). It is therefore not surprising that trust has been reported to correlate positively with Extraversion and Agreeableness traits (Hiraishi et al., 2008). In addition, while Conscientiousness and Openness may be positively related to trustworthiness, Neuroticism has been found to correlate negatively with trust (Evans and Revelle, 2008). Moreover, an inverse relationship between trust and stress is reported in studies of the associated hormones oxytocin (trust) and cortisol (stress, Takahashi et al., 2005; Cardoso et al., 2013; McQuaid et al., 2016), and of how stress-induced participants seek out prosocial interactions including trust (von Dawans et al., 2012; Raposa et al., 2015). Similarly, higher stress may lead to less trusting decision-making (FeldmanHall et al., 2015). In addition, it has been confirmed in both neuroimaging experiments (Yanagisawa et al., 2011) and experiments that when faced with stressors and threats people may upregulate their level of trust (Koranyi and Rothermund, 2012), possibly as a way of coping. Positive relationships have also found between trust and active appraisal and coping strategies (Buchwald, 2003; Wilson and Darke, 2012).

In trust research, another approach is to observe levels of trust in general within and between social groups. Such general trust (sometimes referred to as social capital) is generally considered important for society at large. It has been reported to be associated with general well-being (Carl and Billari, 2014), well-functioning democracies (Uslaner, 2002), economic growth (Guiso et al., 2004), cooperation (King-Casas et al., 2005), intelligence (Hooghe et al., 2012), and health (Kawachi et al., 2008; Giordano and Lindström, 2016; Giordano et al., 2019). Trust is thus associated with several beneficial outcomes that may function as buffers to adverse stressors. Resilience to stress has been described by Folkman and Lazarus (1985) as a dynamic coping process that involves efforts to face external threats and demands. Research on coping has traditionally focused on reactive strategies, e.g., "problem-focused coping" and "emotion-focused coping" (Lazarus and Folkman, 1984). Other approaches such as proactive and preventive coping (e.g., Schwarzer and Taubert, 2002) are more goal-oriented in creating a mental preparedness for potentially stressful events. Proactive coping is assumed to promote a positive mood and an enhanced focus on future events that may alleviate psychopathological outcomes (Greenglass and Fiksenbaum, 2009). While most measurement instruments regard coping as a reactive process (e.g., Kato, 2013), the Proactive Coping Inventory (PCI), which is used in the present research (Greenglass and Fiksenbaum, 2009), represents a more forward-oriented outlook, measuring characteristics such as self-regulation and goal attainment. The PCI measure has been found to also correlate with the personality traits measured in the FFM of personality (Costa and McCrae, 1992); in particular, Extraversion and Conscientiousness appear to be positively related to PCI, whereas Neuroticism shows a negative relationship (Straud et al., 2015).

As reviewed above, while there is some evidence that general trust is beneficial for health (Kawachi et al., 2008; Giordano and Lindström, 2016; Giordano et al., 2019), there is a need to also investigate contextual effects and similar constructs of trust in order to explain and evaluate the efficacy and relevance of trust as a health-promoting factor (Shiell et al., 2020). In line with the stress-buffering hypothesis of general trust (Cohen and Wills, 1985; McQuaid et al., 2016), we apply an eclectic approach, in order to deepen our understanding of the healthpromoting effects of general trust (Shiell et al., 2020). We do this by examining how individual differences in general trust, proactive coping, personality, and IU jointly relate to perceived stress. In two representative American community samples, we test the statistical relations between the measurements of these constructs, while controlling for background variables (age, gender, education, and income). To our knowledge, few studies have investigated how such constructs jointly relate to, drive and possibly alleviate perceived stress in general population samples. We question whether a partly dispositional measure of general trust (GTS) and a more context sensitive measure of proactive coping (PCI) can explain variance in perceived stress (PSS) over and above what background variables [socioeconomic status (SES)], personality (HEXACO), and intolerance of uncertainty (IUS) can explain.

\section{STUDY 1}

\section{Participants and Procedure}

Participants partaking in Study $1(N=1,213)$ were recruited from Qualtrics Research Panels in June 2018. The design of the study was a cross-sectional survey programmed in the online survey platform Qualtrics XM. Qualtrics provides access to a pool of online panels that can be recruited to partake in research studies. This type of data collection method is becoming increasingly popular and the data quality from the Qualtrics panel seems to be on par with data from conventional data collection methods (e.g., Walter et al., 2019). A Qualtrics project manager invited panel participants as needed using quota sampling in order to achieve a sample of participants residing in the United States where the sample had the same proportion as the American population based on gender $(51.2 \%$ female), age $(18-24=12.7 \%, 25-34=17.8 \%, 35-44=16.9 \%$, $45-54=18.1 \%, 55-64=16.2 \%, 65+=18.2 \%)$, education (bachelor's degree or higher $=33.5 \%$ ), and income (US median income or less $=44.1 \%$ ). This sampling procedure entails a non-probabilistic stratification, in that participants are continually invited until the pre-defined quota, for example between males and females, is met. There were no missing data in the dataset since we used firstly response requests and secondly forced responses for each question. The identity of the participants is not known to us but technically the identities of the participants may be seen as a pseudonymized form of de-identification since Qualtrics has a theoretical possibility to link a response ID with a living person.

\section{Measures}

The PSS scale has been validated in a number of languages and cultures (Lee, 2012) and is shown to be related to biological stress markers (Epel et al., 2004). The PSS originally consisted of 14 items (PSS-14) measuring perceived stress but was later 
reduced to 10 items (PSS-10) due to improved psychometric properties (Cohen and Williamson, 1988) and is therefore recommended for both practice and research (Lee, 2012). The PSS-14 (Cohen et al., 1983) contains 14 items that ask respondents about their feelings and thoughts during the last month. Each statement is phrased using how often and is rated on a fivepoint scale ranging from 0 ("never") to 4 ("very often," e.g., "In the last month, how often have you felt nervous and 'stressed'?"). The PSS-14 score is commonly obtained by summing all items to a total perceived stress score. We collected data for the 14 items but we used the 10-item version (PSS-10; Cohen and Williamson, 1988) in the analyses.

Intolerance of Uncertainty Scale was developed as an instrument comprising 27 items measuring dispositional sensitivity for uncertain events (Freeston et al., 1994), and was later translated (Buhr and Dugas, 2002) and shortened to a 12-item version (Carleton et al., 2007) with reliable psychometric properties (Hale et al., 2016). The IUS-12 (Carleton et al., 2007) consists of 12 statements about subjective responses to uncertain events. The IUS-12 comprises two factors: prospective anxiety (e.g., "I always want to know what the future has in store for me") and inhibitory anxiety (e.g., "When it's time to act, uncertainty paralyzes me"). Each statement is assessed using a five-point rating scale, ranging from 1 ("Not at all characteristic of me") to 5 ("Entirely characteristic of me"). The scoring of IUS-12 is usually assessed using the total or the sum of the statements pertaining to each of the two factors.

The HEXACO-60 short personality inventory (Ashton and Lee, 2009) assesses the six dimensions of the HEXACO model of personality: Honesty-Humility (H), Emotionality (E), Extraversion (X), Agreeableness (A), Conscientiousness (C), and Openness to experience $(\mathrm{O})$. Each dimension contains 10 statements taken from the original HEXACO-PI-R (e.g., "If I want something from someone, I will laugh at that person's worst jokes"). Each statement is rated on a five-point scale ranging from 1 ("strongly disagree") to 5 ("strongly agree").

General trust is often measured in surveys such as the American General Social Survey (GSS) using a single-item question. Yamagishi and Yamagishi (1994) proposed a six-item scale, the GTS, based on several other scales. GTS has been validated across domains and in different languages, and the most recent version is a revised and shortened five-item scale, which is used in the present study (Yamagishi et al., 2015). Each statement of the GTS scale (e.g., "Generally, I trust others") is rated on a five-point scale ranging from 1 ("completely disagree") to 5 ("completely agree").

\section{Assumptions and Data Analytic Plan}

All included constructs are computed as aggregate variables according to each instrument's scoring. We provide descriptive statistics for each variable including Mean, Standard Deviation, Skewness, Kurtosis, Cronbach's $\alpha$, and a Pearson correlation table, showing how each variable is related in terms of bi-variate linear correlation.

Broadly, we assume that the instruments used as predictors are confounded and thus may overlap statistically in explaining psychological stress (PSS). A set of more specific assumptions will guide how we approach the data in the following steps. Trait personality theory consists of broader and narrower constructs often hierarchically ordered (e.g., Soto and John, 2017). It is widely accepted that higher-order constructs and processes develop or manifest into lower-order traits followed by even lower-order specific actions and strategies. Hierarchically higher-order traits are less context-dependent and more related to neurobiological underpinnings, whereas lower-order facets are more contextdependent in relation to specific situations and behaviors (DeYoung, 2010, 2013). We will apply a hierarchical approach between, as opposed to within, different constructs when modeling the data.

While Sexton et al. (2003) have modeled personality traits as a higher-order construct compared to IUS and PSS, Hiraishi et al. (2008) specify general trust as a lower-order construct compared to personality. We, therefore, assume that HEXACO is a higher-order construct compared to IUS, GTS, and PSS. We accordingly model them from higher to lower order in a hierarchical multiple regression analysis (e.g., Tabachnick and Fidell, 2013), in that a higher-order construct will precede a lower-order construct in the equation. The control variables of SES are however entered first.

We expect IUS and Emotionality (E) to be positively related to PSS, whereas the other personality traits likely are negatively related to PSS (e.g., Carver and Connor-Smith, 2010). Further, we expect that the bi-variate relationship between GTS and PSS will be weaker when modeled hierarchically together with the higher-order constructs of HEXACO and IUS. We furthermore expect that controlling for SES (Age, Gender, Education, and Income) will influence the effects of IUS, HEXACO, and GTS on PSS based on the assumption that education and income also have the potential to alleviate perceived stress (Redmond et al., 2013; Algren et al., 2018).

\section{Results}

As may be seen in Table 1, PSS shows, as expected, positive significant correlations with IUS and Emotionality (E). In addition, Extraversion (X) shows a stronger negative correlation to PSS compared to Honesty-Humility (H), Agreeableness (A), and Conscientiousness $(\mathrm{C})$. Furthermore, Openness $(\mathrm{O})$ shows a weak but significant correlation with PSS, while the other pro-social personality traits $(\mathrm{H}, \mathrm{A}, \mathrm{C})$ and GTS show negative correlations.

In order to examine the expectations described above, we conducted a four-step hierarchical regression analysis. As may be seen in Table 2, in the first step, four SES-variables (Age, Gender, Education, and Income) are entered together accounting for $19 \%$ of the variance in PSS. All SES-variables show unique and significant associations in that higher age, higher income, and higher education as well as male gender are all associated with lower PSS. The second step reveals that HEXACO adds $29 \%$ of the explained variance in PSS. Jointly with SES, nearly half of the variance in PSS is now explained. Each factor in the HEXACO model is a significant predictor of PSS, where $\mathrm{E}$ and $\mathrm{O}$ indicate positive relationships and the other factors indicate negative relationships to PSS. As expected, Emotionality and Extraversion show the strongest associations. In the third step, the inclusion of IUS shows a significant and positive relationship with PSS and adds 3\% of the uniquely 
TABLE 1 I Means (M), standard deviation (SD), skewness (Sk), kurtosis (Ku) and Pearson correlations of study variables.

\begin{tabular}{|c|c|c|c|c|c|c|c|c|c|c|c|c|c|}
\hline Variables & $M$ & $S D$ & Sk & $\mathrm{Ku}$ & 1 & 2 & 3 & 4 & 5 & 6 & 7 & 8 & 9 \\
\hline 1. PSS-10 & 1.72 & 0.74 & 0.11 & 3.07 & 0.89 & & & & & & & & \\
\hline 2. IUS-12 & 2.67 & 0.74 & 0.40 & 2.87 & $0.47^{* * * *}$ & 0.87 & & & & & & & \\
\hline 3. $\mathrm{H}$ & 3.55 & 0.62 & -0.09 & 2.76 & $-0.25^{* * *}$ & $-0.22^{* * * *}$ & 0.73 & & & & & & \\
\hline 4. E & 3.18 & 0.61 & -0.11 & 3.41 & $0.48^{* * * *}$ & $0.43^{* * * *}$ & -0.01 & 0.75 & & & & & \\
\hline 5. $x$ & 3.18 & 0.67 & -0.40 & 3.38 & $-0.53^{* * *}$ & $-0.33^{* * *}$ & $0.10^{* * *}$ & $-0.35^{* * *}$ & 0.81 & & & & \\
\hline 6. A & 3.22 & 0.59 & -0.22 & 3.36 & $-0.36^{* * *}$ & $-0.23^{* * * *}$ & $0.33^{* * *}$ & $-0.16^{* * *}$ & $0.36^{* * * *}$ & 0.75 & & & \\
\hline 7. C & 3.68 & 0.59 & -0.21 & 2.61 & $-0.34^{* * *}$ & $-0.15^{* * *}$ & $0.40^{* * *}$ & $-0.12^{* * *}$ & $0.33^{* * *}$ & $0.26^{* * * *}$ & 0.78 & & \\
\hline 8. 0 & 3.33 & 0.66 & -0.12 & 2.96 & $-0.08^{* *}$ & $-0.11^{* * * *}$ & $0.06^{*}$ & $-0.10^{* * *}$ & $0.31^{* * * *}$ & $0.19^{* * * *}$ & $0.27^{* * * *}$ & 0.78 & \\
\hline 9. GTS & 3.27 & 0.82 & -0.43 & 2.89 & $-0.36^{* * *}$ & -0.11 & $0.08^{* *}$ & $-0.14^{* * *}$ & $0.38^{* * * *}$ & $0.29^{* * * *}$ & $0.13^{* * *}$ & $0.13^{* * *}$ & 0.90 \\
\hline
\end{tabular}

Internal consistency reliabilities (Cronbach's $\alpha$ ) in bold in the main diagonal. An extended version of the correlations including subscales of IUS-12 as well as factors and facets of HEXACO is available in the Supplementary Material. PSS-10, Perceived Stress Scale (short 10-tem version); IUS-12, Intolerance of Uncertainty Scale (short 12-item version); H, Honesty Humility (60-item HEXACO-PI-R (HEXACO-60); E, Emotionality (60-item HEXACO-PI-R (HEXACO-60); X, EXtraversion (60-item HEXACO-PI-R (HEXACO-60); A, Agreeableness (60-item HEXACO-PI-R (HEXACO-60); C, Conscientiousness (60-item HEXACO-PI-R (HEXACO-60); O, Openness (60-item HEXACO-PI-R (HEXACO-60); GTS, General Trust Scale (revised 5-item version). * $p<0.05 ;{ }^{* *} p<0.01 ;{ }^{* * *} p<0.001$.

TABLE 2 | Hierarchical multiple regression model testing effects on the Perceived Stress Scale (PSS-10).

\begin{tabular}{|c|c|c|c|c|c|c|c|c|}
\hline \multirow[t]{3}{*}{ Variables } & \multicolumn{8}{|c|}{ Model $^{a}$} \\
\hline & \multicolumn{2}{|c|}{1} & \multicolumn{2}{|c|}{2} & \multicolumn{2}{|c|}{3} & \multicolumn{2}{|c|}{4} \\
\hline & $\beta$ & $S E$ & $\beta$ & SE & $\beta$ & SE & $\beta$ & $S E$ \\
\hline $\mathrm{H}$ & & & $-0.09^{* * * *}$ & 0.03 & $-0.06^{*}$ & 0.03 & $-0.06^{* *}$ & 0.03 \\
\hline$E$ & & & $0.31^{* * *}$ & 0.03 & $0.23^{* * *}$ & 0.03 & $0.24^{* * *}$ & 0.03 \\
\hline$x$ & & & $-0.30^{* * * *}$ & 0.03 & $-0.26^{* * *}$ & 0.03 & $-0.23^{* * *}$ & 0.03 \\
\hline$A$ & & & $-0.13^{* * * *}$ & 0.03 & $-0.12^{* * * *}$ & 0.03 & $-0.10^{* * * *}$ & 0.03 \\
\hline C & & & $-0.12^{* * *}$ & 0.03 & $-0.13^{* * *}$ & 0.03 & $-0.14^{* * *}$ & 0.03 \\
\hline $\mathrm{O}$ & & & $0.11^{* * *}$ & 0.03 & $0.11^{* * * *}$ & 0.02 & $0.11^{* * * *}$ & 0.02 \\
\hline IUS-12 & & & & & $0.19^{* * *}$ & 0.02 & $0.20^{* * * *}$ & 0.02 \\
\hline GTS & & & & & & & $-0.13^{* * *}$ & 0.02 \\
\hline Age & $-0.28^{* * * *}$ & 0.01 & $-0.15^{\text {**** }}$ & 0.01 & $-0.12^{* * *}$ & 0.01 & $-0.11^{* * *}$ & 0.01 \\
\hline Gender $^{b}$ & $0.12^{* * *}$ & 0.04 & -0.01 & 0.04 & 0.01 & 0.04 & 0.01 & 0.03 \\
\hline Education & $-0.09^{* *}$ & 0.01 & -0.02 & 0.01 & -0.03 & 0.01 & -0.01 & 0.01 \\
\hline Income & $-0.15^{* * * *}$ & 0.01 & $-0.08^{* *}$ & 0.01 & $-0.08^{* * *}$ & 0.01 & $-0.08^{* *}$ & 0.01 \\
\hline$A d j^{R 2}$ & $0.19^{* * * *}$ & & $0.48^{* * * *}$ & & $0.51^{\text {**** }}$ & & $0.52^{* * *}$ & \\
\hline$\triangle A d j^{R 2}$ & & & $0.29^{* * * *}$ & & $0.03^{* * * *}$ & & $0.01^{* * * *}$ & \\
\hline$F$ & $71.52^{* * * *}$ & & $114.78^{* * * *}$ & & $116.08^{* * * *}$ & & $112.42^{* * * *}$ & \\
\hline
\end{tabular}

PSS-10, Perceived Stress Scale (short 10-tem version); IUS-12, Intolerance of Uncertainty Scale (short 12-item version); H, Honesty Humility (60-item HEXACO-PI-R (HEXACO-60); E, Emotionality (60-item HEXACO-PI-R (HEXACO-60); X, Extraversion (60-item HEXACO-PI-R (HEXACO-60); A, Agreeableness (60-item HEXACO-PI-R (HEXACO-60); C, Conscientiousness (60-item HEXACO-PI-R (HEXACO-60); O, Openness (60-item HEXACO-PI-R (HEXACO-60); GTS, General Trust Scale (revised 5-item version).

${ }^{a}$ Perceived Stress Scale (PSS-10) modeled as dependent variable. ${ }^{b}$ Female $=1$.

${ }^{*} p<0.05 ;{ }^{* *} p<0.01 ;{ }^{* * *} p<0.001$.

explained variance with slightly attenuated coefficients of Emotionality and Extraversion. When GTS is included in the fourth and final step, the explained variance is only marginally improved. Still, GTS shows a unique and significant negative relation to PSS when all other variables are controlled for.

\section{Discussion}

These results indicate firstly, that the bi-variate relationships displayed in Table 1 can be disentangled and to some degree explained using a hierarchical regression model. Secondly, the assumed higher-order HEXACO construct (Sexton et al., 2003) contributes the most toward explaining PSS, especially the traits of Emotionality (cf. Neuroticism) and Extraversion, which is in line with previous research (Carver and Connor-Smith, 2010; Kotov et al., 2010). Furthermore, although IUS is commonly related to worry and anxiety (Carleton, 2016), the results show that IUS and PSS are associated, as have been reported in other studies as well (Crum et al., 2013). According to McEvoy and Mahoney (2012), the construct of IU should theoretically be seen as distinct from personality and more specifically the trait of Neuroticism. However, the results above indicate that the relationship between IUS and PSS is attenuated when modeled together with HEXACO. Moreover, IUS remains a stronger predictor of PSS compared to GTS, which may support the notion that IU is a higher ordered construct compared to trust (Hiraishi et al., 2008). Although the coefficients are relatively small in the regression compared to the bi-variate correlations, IUS and GTS add significantly to the explained variance of PSS, even after controlling for strong predictors such as personality and socioeconomic background. We, therefore, see the observed effects of IU and general trust as important predictors of psychological stress. 
Since we assumed the tested constructs to be inter-correlated it should be noted that each incremental step in the hierarchical regression was expected to increase explained variance. It should also be acknowledged that predictors entered first in a multiple linear regression which generally tend to account for the largest share of variance. It is, therefore important to consider the possible hierarchical order between the constructs and we have provided a rationale for why higher-order constructs such as personality to some extent could account for the variance in lower-order constructs like IU or trust in predicting perceived stress.

\section{STUDY 2}

In Study 2, we move forward by once again testing the potentially alleviating effects of trust, this time however without HEXACO in the model. Instead, we decided to include a multi-dimensional but lower-order measure of proactive coping, PCI (see Section Measures and Assumptions and Data Analytic Plan for details).

\section{Participants and Procedure}

Participants partaking in Study $2(N=1,090)$ were recruited from Qualtrics Research Panels in November 2018. The procedure for Study 2 was practically identical as in Study 1. The study was a cross-sectional design using quota sampling in order to achieve a sample of participants residing in the United States that had the same proportions as the American population based on gender $(53.9 \%$ female), age $(18-24=12.3 \%$, $25-34=18.7 \%, 35-44=17.7 \%, 45-54=18.3 \%, 55-64=15.9 \%$, $65+=17.1 \%$ ), education (bachelor's degree or higher $=33.3 \%$ ), and income (US median income or less $=45.9 \%$ ).

\section{Measures}

Similar to Study 1, the participants responded to the PSS-14 (Cohen et al., 1983), the IUS-12 (Carleton et al., 2007), and the GTS (Yamagishi et al., 2015; see Section Measures in Study 1). We also included the PCI measure (Greenglass et al., 1999) consisting of 55 items using a four-point rating scale ranging from 1 ("Not true at all") to 4 ("completely true"). Greenglass et al. (1999) developed the multidimensional PCI consisting of the seven subscales: Proactive Coping Scale (PCS), Reflective Coping Scale (RCS), Strategic Planning Scale (SPS), Preventive Coping Scale (PrCS), Instrumental Support Seeking Scale (ISSS), Emotional Support Seeking Scale (EMSS), and Avoidance Coping Scale (ACS). These subscales partly overlap and their precise factor structures have been questioned (Roesch et al., 2009), but the two fundamental factors of proactive (PCS) and preventive coping (PrCS) seem to represent distinct and unidimensional constructs (Drummond and Brough, 2016). The PCS measures goal attainment, cognition, and behavior (e.g., "I visualize my dreams and try to achieve them"); the RCS describes contemplation and simulation of possible future events (e.g., "I imagine myself solving a difficult problem before I actually have to face it"); the SPS focuses on creating a goal-oriented plan of manageable action (e.g., "I break down a problem into smaller parts and do one part at a time"); the PrCS measures the level of anticipation and preparedness (e.g., "I think ahead to avoid dangerous situations"); the ISSS measures advice or feedback from one's immediate social network (e.g., "I ask others what they would do in my situation"); the ESSS captures the desire to share one's feelings in order to establish an alliance of empathy and comfort (e.g., "When I'm depressed I get out and talk to others"); and the ACS describes passive actions that seek to elude stressful events (e.g., "When I have a problem I like to sleep on it").

\section{Assumptions and Data Analytic Plan}

Since Study 1 showed that GTS correlated significantly with all facets of HEXACO (see Table 1) and that personality could explain $29 \%$ of the variance in PSS (see Table 2 ), we decided to now model GTS together with SES, IUS, and the multidimensional PCI measure, this time however without the HEXACO measure in the model. The effects of potentially important coping strategies are now tested using PCI (the Proactive Coping Inventory, consisting of the seven subscales; Greenglass et al., 1999). It is reasonable to assume that GTS will be positively related to PCI due to the similarities and possible relationship with social support. Furthermore, PCI has been reported to promote a positive mood (Schwarzer and Taubert, 2002) and alleviate psychopathological outcomes (Greenglass and Fiksenbaum, 2009). On an aggregated level, we believe that PCI will be negatively related to PSS. It remains to be seen which of the specific PCI subscales may predict PSS.

Since we firstly see GTS as rather similar to the A trait and, secondly, believe that specific proactive coping strategies and behaviors may be learned and depend on situations, we treat PCI as a lower-ordered construct compared to GTS. Consequently, PCI is added in the fourth and last hierarchical steps. We conjecture that the relationship between GTS and PSS may be weaker when modeled hierarchically foremost together with the higher-order construct of IUS, but also together with the lower-ordered construct of PCI. We furthermore expect that controlling for SES (Age, Gender, Education, and Income) will influence the effects of IUS, GTS, and PCI on PSS, based on the assumption that education and income also have the potential to alleviate perceived stress (Redmond et al., 2013; Algren et al., 2018).

All the measured constructs are entered in a hierarchical regression model. SES is entered first, IUS in the second, and GTS in the third step. Since specific proactive coping strategies and behaviors may be learned and GTS is similar to the A factor, PCI is here assumed to be a lower-order construct compared to GTS. Consequently, PCI is added in the fourth and last step of the model.

\section{Results}

As may be seen in Table 3, the bi-variate relations from Study 1 are replicated, as a positive significant correlation is found between PSS and IUS and a negative significant correlation between GTS and PSS. The subscales of PCI display rather strong significant negative correlations to PSS, with the exceptions 
TABLE 3 I Means (M), standard deviation (SD), skewness (Sk), kurtosis (Ku) and Pearson correlations of study variables.

\begin{tabular}{|c|c|c|c|c|c|c|c|c|c|c|c|c|c|c|}
\hline Variables & $M$ & $S D$ & Sk & $\mathrm{Ku}$ & 1 & 2 & 3 & 4 & 5 & 6 & 7 & 8 & 9 & 10 \\
\hline $\begin{array}{l}\text { 1. PSS- } \\
10\end{array}$ & 1.76 & 0.74 & -0.03 & 2.69 & 0.88 & & & & & & & & & \\
\hline 2. IUS-12 & 2.65 & 0.82 & 0.43 & 2.76 & $0.53^{* * * * *}$ & 0.90 & & & & & & & & \\
\hline 3. PCS & 2.97 & 0.48 & -0.47 & 3.44 & $-0.33^{* * * *}$ & $-0.13^{* * * *}$ & 0.86 & & & & & & & \\
\hline 4. RCS & 3.02 & 0.55 & -0.65 & 3.94 & $-0.15^{* * * *}$ & $0.12^{* * * *}$ & $0.65^{* * * *}$ & 0.89 & & & & & & \\
\hline 5. SPS & 2.99 & 0.62 & -0.59 & 3.43 & $-0.19^{* * * *}$ & $0.10^{* *}$ & $0.59^{* * * *}$ & $0.69^{* * * *}$ & 0.76 & & & & & \\
\hline 6. PrCS & 2.94 & 0.59 & -0.60 & 3.37 & $-0.26^{* * * *}$ & 0.06 & $0.62^{* * * *}$ & $0.71^{* * *}$ & $0.67^{* * * *}$ & 0.88 & & & & \\
\hline 7. ISSS & 2.83 & 0.65 & -0.42 & 2.97 & -0.01 & $0.13^{* * * * *}$ & $0.44^{* * * *}$ & $0.52^{* * * *}$ & $0.49^{* * * *}$ & $0.52^{* * * *}$ & 0.90 & & & \\
\hline 8. ESSS & 2.78 & 0.76 & -0.37 & 2.51 & $-0.20^{* * *}$ & -0.02 & $0.44^{* * *}$ & $0.44^{* * *}$ & $0.45^{* * * *}$ & $0.47^{* * * *}$ & $0.71^{* * * *}$ & 0.84 & & \\
\hline 9. ACS & 2.63 & 0.70 & -0.26 & 2.88 & $0.07^{*}$ & $0.16^{* * * *}$ & -0.02 & $0.21^{* * * *}$ & $0.14^{* * * *}$ & $0.14^{* * * *}$ & $0.20^{* * * *}$ & $0.14^{* * * *}$ & 0.66 & \\
\hline 10. GTS & 3.23 & 0.80 & -0.43 & 3.10 & $-0.28^{* * * *}$ & -0.13 & $0.21^{* * * *}$ & $0.22^{* * * *}$ & $0.24^{* * * *}$ & $0.26^{* * * *}$ & $0.24^{* * * *}$ & $0.28^{* * * *}$ & $0.13^{* * * *}$ & 0.88 \\
\hline
\end{tabular}

Internal consistency reliabilities (Cronbach's $\alpha$ ) in bold in the main diagonal. PSS-10, Perceived Stress Scale (short 10-tem version); IUS-12, Intolerance of Uncertainty Scale (short 12item version); PCS, Proactive Coping Scale (Proactive Coping Inventory); RCS, Reflective Coping Scale (Proactive Coping Inventory); SPS, Strategic Planning Scale (Proactive Coping Inventory); PrCS, Preventive Coping Scale (Proactive Coping Inventory); ISSS, Instrumental Support Seeking Scale (Proactive Coping Inventory); ESSS, Emotional Support Seeking Scale (Proactive Coping Inventory); ACS, Avoidance Coping Scale (Proactive Coping Inventory); GTS, General Trust Scale (revised 5-item version). ${ }^{*} p<0.05 ;{ }^{* *} p<0.01$; **** $p<0.001$.

of the uncorrelated SSS and the weakly positively correlated ACS. Furthermore, IUS shows relatively small correlations with the subscales of PCI. It should be noted that there are positive significant correlations between GTS and each subscale of PCI, suggesting that the more people engage in proactive coping the higher the levels of trust is observed and vice versa.

As in Study 1, a four-step hierarchical regression was conducted. As may be seen in Table 4, the effects of the SES variables in step 1 are similar to the results in Study 1, as age, gender, education, and income account for $18 \%$ of the variance in PSS. Again, higher age, income, education, and reporting a male gender are associated with lower PSS. In step 2, IUS shows a strong significant association to PSS and the explained variance increases to $36 \%$ jointly with SES. This effect of IUS remains in the third step, whereas GTS shows a significant inverse relationship with PSS similar to Study 1. In the fourth and final step, IUS remains a strong predictor of PSS and the seven subscales of PCI add an additional 9\% of explained variance. Jointly, SES, IUS, GTS, and PCI now explain $47 \%$ of the variance in PSS. The PCI subscales show some overlap in the regression but the Variance Inflation Factors $(\mathrm{VIF}<3)$ do not indicate severe multicollinearity (Hair et al., 2014). The subscales PCS, PrCS, and ESSS show significant inverse effects on PSS, whereas ISSS interestingly indicate a positive effect, when all of the other variables are accounted for, in contrast to the non-significant pairwise correlation between ISSS and PSS. As expected, the effect of GTS is attenuated when modeled together with PCI. While the IUS construct explains the most variance, the lower-ordered PCI adds significantly to the explained variance in PSS. As in Study 1, the explanatory power of GTS is low, but significant and in the expected inverse direction.

\section{Discussion}

We conclude that, in contrast to Study 1, IUS now shows a stronger relationship to PSS and explains a larger part of the variance in PSS and that this association largely is in line with previously reported studies (Crum et al., 2013). Since the GTS construct again adds significantly to the explained variance of PSS even after controlling for strong factors such as intolerance of uncertainty and socio-economic background, we conclude that trust shows a unique, albeit small, effect under control for interrelated constructs. However, the results indicate that the predictive power of trust on perceived stress is attenuated when proactive coping is introduced. This is not surprising since the PCI subscales contain two accounts of social support, different behaviors, and coping strategies, that involve respective others and social connectedness to a large degree. The PCI seems to add a unique effect contributing to perceived stress above and beyond the effects of SES, IUS, and GTS. An inverse association between PSS and a coping inventory such as PCI should be expected (Schwarzer and Taubert, 2002; Greenglass and Fiksenbaum, 2009). Additionally, the PCI has been reported to be positively related to the personality trait of $\mathrm{E}$ and $\mathrm{C}$ and negatively related to Neuroticism (Straud et al., 2015). In a similar fashion, $\mathrm{C}$ and $\mathrm{O}$ have been reported to be positively related to trustworthiness while Neuroticism has been found to correlate negatively with trust (Evans and Revelle, 2008). It is thus suggested that proactive coping and trust may be comparable in terms of their associations and possibly predictive validity in explaining stress-related outcomes.

Although we acknowledge that the hierarchical order in which the constructs of GTS and PCI theoretically could be structured is up for debate, we interpret our results as suggesting that trust or social support can be understood as a subset of proactive coping. Yet, the many different subscales of the PCI should be expected to account for more variance since it consists of more coping constructs than social support. All subscales of PCI in fact show significant inverse associations to PSS with the interesting exception of ISSS which relates positively to stress. This may reflect that the subscales of PCI are conditional on each other or that one of the subscales acts as a suppressor variable in the regression (e.g., MacKinnon et al., 2000). Overall, the results from Study 2 largely confirm the patterns from Study 1 in that trust, with the addition of proactive coping, can reduce stress over and above what can be explained by personality and socio-economic variables. 
TABLE 4 | Hierarchical multiple regression model testing effects on the Perceived Stress Scale (PSS-10).

\begin{tabular}{|c|c|c|c|c|c|c|c|c|}
\hline \multirow[t]{3}{*}{ Variables } & \multicolumn{8}{|c|}{ Model $^{\mathbf{a}}$} \\
\hline & \multicolumn{2}{|c|}{1} & \multicolumn{2}{|c|}{2} & \multicolumn{2}{|c|}{3} & \multicolumn{2}{|c|}{4} \\
\hline & $\beta$ & $S E$ & $\beta$ & $S E$ & $\beta$ & $S E$ & $\beta$ & $S E$ \\
\hline IUS-12 & & & $0.45^{* * * *}$ & 0.02 & $0.44^{* * * *}$ & 0.02 & $0.41^{* * * * *}$ & 0.02 \\
\hline GTS & & & & & $-0.14^{* * *}$ & 0.02 & $-0.08^{* *}$ & 0.02 \\
\hline PCS & & & & & & & $-0.14^{* * * *}$ & 0.05 \\
\hline RCS & & & & & & & 0.03 & 0.05 \\
\hline SPS & & & & & & & -0.05 & 0.04 \\
\hline PrCs & & & & & & & $-0.17^{* * * *}$ & 0.04 \\
\hline ISSS & & & & & & & $0.22^{* * * *}$ & 0.04 \\
\hline ESSS & & & & & & & $-0.18^{* * * *}$ & 0.03 \\
\hline ACS & & & & & & & 0.02 & 0.03 \\
\hline Age & $-0.34^{* * * *}$ & 0.01 & $-0.21^{* * * *}$ & 0.01 & $-0.18^{* * *}$ & 0.01 & $-0.19^{* * * *}$ & 0.01 \\
\hline Gender $^{\mathrm{b}}$ & $0.15^{* * * *}$ & 0.04 & $0.12^{* * * *}$ & 0.04 & $0.11^{* * * *}$ & 0.04 & $0.12^{* * *}$ & 0.04 \\
\hline Education & -0.05 & 0.02 & $-0.06^{*}$ & 0.01 & -0.03 & 0.01 & 0.01 & 0.01 \\
\hline Income & $-0.09^{* * *}$ & 0.01 & $-0.08^{* *}$ & 0.01 & $-0.07^{*}$ & 0.01 & -0.05 & 0.01 \\
\hline$A d j^{R 2}$ & $0.18^{* * * *}$ & & $0.36^{* * * *}$ & & $0.38^{* * * *}$ & & $0.47^{* * * *}$ & \\
\hline$\Delta A d j^{R 2}$ & & & $0.18^{* * *}$ & & $0.02^{* * * *}$ & & $0.09^{* * *}$ & \\
\hline$F$ & $60.83^{* * * *}$ & & $124.88^{* * *}$ & & $112.29^{* * * *}$ & & $74.76^{* * * *}$ & \\
\hline
\end{tabular}

PSS-10, Perceived Stress Scale (short 10-tem version); IUS-12, Intolerance of Uncertainty Scale (short 12-item version); PCS, Proactive Coping Scale (Proactive Coping Inventory); RCS, Reflective Coping Scale (Proactive Coping Inventory); SPS, Strategic Planning Scale (Proactive Coping Inventory); PrCS, Preventive Coping Scale (Proactive Coping Inventory); ISSS, Instrumental Support Seeking Scale (Proactive Coping Inventory); ESSS, Emotional Support Seeking Scale (Proactive Coping Inventory); ACS, Avoidance Coping Scale (Proactive Coping Inventory); GTS, General Trust Scale (revised 5-item version).

${ }^{a}$ Perceived Stress Scale (PSS-10) modeled as dependent variable. ${ }^{b}$ Female $=1$.

${ }^{*} p<0.05 ;{ }^{* *} p<0.01 ;{ }^{* * *} p<0.001$.

\section{GENERAL DISCUSSION}

In two studies, we model how the constructs of general trust (GTS), proactive coping (PCI, in Study 2), personality (HEXACO, in Study 1), and intolerance of uncertainty (IUS), jointly relate to perceived stress (PSS) while controlling for SES. Our aim is to investigate whether a measure of general trust (GTS) and a measure of proactive coping (PCI) can explain variance in perceived stress (PSS) over and above what background variables (SES), personality (HEXACO), and IU (IUS) can explain. We model these constructs hierarchically based on the assumption that there are higher-order constructs that are less context-dependent and more related to neurobiological underpinnings and lower-order constructs that are more context-dependent in relation to specific situations and behaviors (DeYoung, 2010, 2013).

In line with the stress-buffering hypothesis of social support (Cohen and Wills, 1985), we suggest that general trust may function as a coping mechanism in alleviating psychological stress. In both our studies, we find the expected negative relationship between general trust and perceived stress. A number of studies substantiate this finding in showing that trust and stress are inversely related (Takahashi et al., 2005; Cardoso et al., 2013; FeldmanHall et al., 2015; McQuaid et al., 2016) and that stress may lead people to seek out more prosocial interactions (von Dawans et al., 2012; Raposa et al., 2015). Other studies have furthermore shown positive relations between trust and active appraisal and coping strategies (Buchwald, 2003; Wilson and Darke, 2012).

Given this, there is a case to be made for the reactivity of trust as a coping mechanism in that when faced with stressful situations, individuals as a response seem to upregulate their level of trust (Yanagisawa et al., 2011; Koranyi and Rothermund, 2012). The positive association between general trust and proactive coping found in the present research is therefore not surprising, especially since the proactive coping inventory in part consists of social support. Yet, it was not clear from the onset of this research how the subscales of proactive coping would be related to general trust. Our findings show that general trust was significantly correlated with each subscale and that the ACS showed the weakest relationship, which is in line with the idea that higher trust is related to more engaged coping strategies (Buchwald, 2003; Wilson and Darke, 2012).

In light of our specified confounding expectation, we find support for the notion that the effect of general trust on perceived stress is attenuated when modeled hierarchically with other interrelated constructs such as the personality inventory of HEXACO, the dispositional construct of IU, the proactive coping inventory of PCI and SES. It is wellknown that personality relates to stress sensitivity along with other psychopathologies (Carver and Connor-Smith, 2010; Kotov et al., 2010; Ebstrup et al., 2011), and we find in line with such previous research that, in particular, emotional stability is positively and $\mathrm{X}$ is negatively related with perceived stress.

The relation between IU and stress is however researched to a lesser degree, as most studies on IU focus on anxiety and mood disorders (Carleton, 2016). While a study by Crum et al. (2013) reports a positive association between IU and perceived stress, our research is to our knowledge 
novel in modeling this specific relationship together with other interrelated constructs. We find this a bit surprising, firstly since it may be intuitive that uncertainty can lead to both subjective and physiological stress responses (De Berker et al., 2016), and secondly, since IU may be linked to different behavioral outcomes (see Birrell et al., 2011 for a review). In contrast to personality traits, the IU measure (IUS) could thus function as a short and specific instrument for measuring dispositional susceptibility to anxiety and stress.

When interpreting our general findings, we conclude that there are several potential buffers to perceived psychological stress, including SES (Redmond et al., 2013; Algren et al., 2018). Specifically, in Study 1 and Study 2 we show that lower perceived stress is associated with background variables such as reporting a male gender, higher age, education, and income. However, when modeled jointly with the constructs of personality, IU, general trust, and proactive coping, the effects of these robust background variables are found to be attenuated. Given this, we consider the presented regression models as a way of showing non-confounded effects.

The awareness of the positive health effects of trust is increasing in public health and social medicine (Kawachi et al., 2008; Giordano and Lindström, 2016). Recent research has requested that future studies should include similar and interrelated constructs in order to control, explain, and disentangle alleged bi-variate relationships between trust and health (Shiell et al., 2020). In response to these requests, we find the alleviating effects of trust on perceived stress to be small but robust. However, given the relatively weak correlations and small amounts of explained variance found it may be questioned whether trust can be effectively increased through programs and policy measures. In our data, the effects of general trust are to the largest part explained by beneficial combinations of constructs that may be more dispositional. Furthermore, although we find that trust is positively related to all the measured subscales of coping and that the effect of trust is attenuated when coping is introduced in our regression model, it may still be questioned if, and to what degree, general trust really can function as a coping mechanism. Assuming that trust at least partly is a context-sensitive construct, we still conjecture that uncertain situations may likely elicit increased levels of trust in relevant experts, professions, and institutions, thus serving as a coping mechanism. Successfully promoting people's health by reducing psychological stress through increased trust therefore requires knowledge of the interactions between general and situational trust (Han, 2019). Whether such reactions to uncertainty could or should be seen as coping is however still up for debate.

\section{Limitations}

The present results are based on data consisting of two samples taken from a pool of web panels of American citizens provided by Qualtrics. Web panels comprised of self-recruited participants who may suffer from selection bias. However, such biases in population sampling are hard to overcome unless mandatory Census programs are used. Another limitation is that we only use self-report instruments in a cross-sectional design. Therefore, we cannot ascertain how the measured constructs relate, for example, to observer ratings, physiological indicators, behavioral data, or causal patterns across the lifespan.

\section{Future Directions}

Our results indicate that dispositional constructs such as personality and IU contribute largely, and that general trust and proactive coping contribute to a lesser degree, in explaining stress. In order to explore the possible stress benefits of trust, experimental studies conducting pre- and post-measures of stress should investigate the efficacy of stress-prevention measures based on attempts to increase general trust and proactive coping. Another endeavor is to investigate the degree to which general trust and proactive coping strategies can alleviate stress for people with different combinations of personality traits. It has furthermore been suggested that much of the earlier research studying the association between general trust and health outcomes are too simplistic and treat general trust as a natural phenomenon. Instead, the link between general trust and health should be investigated from a wider systems perspective acknowledging that cultural differences may be important in explaining this relationship. The causal mechanisms between general trust, coping, stress, and health are far from clear and need to be further investigated.

\section{Conclusions}

Overall, stress is one of the most critical antecedents of negative health outcomes. Assumed to correlate with lower morbidity and mortality, general trust has increasingly been seen as a quick-fix or buffer to psychological stress. We have shown that the negative relationship between trust and stress is robust but weak and significantly reduced when modeled jointly with personality, proactive coping, and socioeconomic status. Our findings suggest that general trust may, to a large degree, be considered a proxy for a beneficial combination of individual characteristics. When studying general trust as a remedy for stress, it is, therefore, reasonable to question what underlies the often overly optimistic health outcomes associated with general trust.

\section{DATA AVAILABILITY STATEMENT}

The raw data supporting the conclusions of this article will be made available by the authors, without undue reservation.

\section{ETHICS STATEMENT}

Ethical review and approval was not required for the study on human participants in accordance with the local legislation and institutional requirements. The patients/participants provided their written informed consent to participate in this study. 


\section{AUTHOR CONTRIBUTIONS}

$\mathrm{AC}$ has the main responsibility for this manuscript. He, together with L-OJ, conceptualized the idea and planning of the study within the research project for which this manuscript is part of the project. AC conducted the data collection, analysis, and a large part of the writing of this manuscript. L-OJ has contributed largely to theoretical development, the structure of the manuscript, reviewing, and writing. Both the authors contributed to the article and approved the submitted version.

\section{FUNDING}

This manuscript is part of a research project led by P.I. AC. He applied for a national call within humanities and social

\section{REFERENCES}

Algren, M. H., Ekholm, O., Nielsen, L., Ersbøll, A. K., Bak, C. K., and Andersen, P. T. (2018). Associations between perceived stress, socioeconomic status, and health-risk behaviour in deprived neighbourhoods in Denmark: a cross-sectional study. BMC Public Health 18:250. doi: 10.1186/s12889-018-5170-x

APA (2020). Stress in America: 2019. Available at: https://www.apa.org/images/ stress-america-2019_tcm7-264533.pdf (Accessed March 5, 2020).

Ashton, M. C., and Lee, K. (2009). The HEXACO-60: a short measure of the major dimensions of personality. J. Pers. Assess. 91, 340-345. doi: $10.1080 / 00223890902935878$

Birrell, J., Meares, K., Wilkinson, A., and Freeston, M. (2011). Toward a definition of intolerance of uncertainty: a review of factor analytical studies of the intolerance of uncertainty scale. Clin. Psychol. Rev. 31, 1198-1208. doi: 10.1016/j.cpr.2011.07.009

Buchwald, P. (2003). The relationship of individual and communal state-trait coping and interpersonal resources as trust, empathy and responsibility. Anxiety Stress Coping 16, 307-320. doi: 10.1080/1061580031000095452

Buhr, K., and Dugas, M. J. (2002). The intolerance of uncertainty scale: psychometric properties of the English version. Behav. Res. Ther. 40, 931-945. doi: 10.1016/S0005-7967(01)00092-4

Cardoso, C., Ellenbogen, M. A., Serravalle, L., and Linnen, A. -M. (2013). Stress-induced negative mood moderates the relation between oxytocin administration and trust: evidence for the tend-and-befriend response to stress? Psychoneuroendocrinology 38, 2800-2804. doi: 10.1016/j.psyneuen. 2013.05.006

Carl, N., and Billari, F. C. (2014). Generalized trust and intelligence in the United States. PLoS One 9:e91786. doi: 10.1371/journal.pone.0091786

Carleton, R. N. (2016). Into the unknown: a review and synthesis of contemporary models involving uncertainty. J. Anxiety Disord. 39, 30-43. doi: 10.1016/j. janxdis.2016.02.007

Carleton, R. N., Mulvogue, M. K., Thibodeau, M. A., McCabe, R. E., Antony, M. M., and Asmundson, G. J. G. (2012). Increasingly certain about uncertainty: intolerance of uncertainty across anxiety and depression. J. Anxiety Disord. 26, 468-479. doi: 10.1016/j.janxdis.2012.01.011

Carleton, R. N., Norton, M. A. P. J., and Asmundson, G. J. G. (2007). Fearing the unknown: a short version of the intolerance of uncertainty scale. J. Anxiety Disord. 21, 105-117. doi: 10.1016/j.janxdis.2006.03.014

Carver, C. S., and Connor-Smith, J. (2010). Personality and coping. Annu. Rev. Psychol. 61, 679-704. doi: 10.1146/annurev.psych.093008.100352

Charles, S. T., Piazza, J. R., Mogle, J., Sliwinski, M. J., and Almeida, D. M. (2013). The wear and tear of daily stressors on mental health. Psychol. Sci. 24, 733-741. doi: 10.1177/0956797612462222

Cohen, S., Kamarck, T., and Mermelstein, R. (1983). A global measure of perceived stress. J. Health Soc. Behav. 24, 385-396. doi: 10.2307/2136404

Cohen, S., and Williamson, G. (1988). "Perceived stress in a probability sample of the United States" in The social psychology of health: Claremont symposium sciences and was awarded a grant from the Swedish Research Council (2016-03059).

\section{ACKNOWLEDGMENTS}

We are most grateful for generous financial support from the Swedish Research Council (Grant 2016-03059).

\section{SUPPLEMENTARY MATERIAL}

The Supplementary Material for this article can be found online at: https://www.frontiersin.org/articles/10.3389/fpsyg.2020.554962/ full\#supplementary-material on applied social psychology. eds. S. Spacapam and S. Oskamp (Newbury Park: Sage), 31-67.

Cohen, S., and Wills, T. A. (1985). Stress, social support, and the buffering hypothesis. Psychol. Bull. 98, 310-357. doi: 10.1037/0033-2909.98.2.310

Colquitt, J. A., Scott, B. A., and LePine, J. A. (2007). Trust, trustworthiness, and trust propensity: a meta-analytic test of their unique relationships with risk taking and job performance. J. Appl. Psychol. 92, 909-927. doi: 10.1037/0021-9010.92.4.909

Costa, P. T., and McCrae, R. R. (1992). Four ways five factors are basic. Pers. Individ. Differ. 13, 653-665. doi: 10.1016/0191-8869(92)90236-I

Coulston, C. M., Bargh, D. M., Tanious, M., Cashman, E. L., Tufrey, K., Curran, G., et al. (2013). Is coping well a matter of personality? A study of euthymic unipolar and bipolar patients. J. Affect. Disord. 145, 54-61. doi: 10.1016/j.jad.2012.07.012

Crum, A. J., Salovey, P., and Achor, S. (2013). Rethinking stress: the role of mindsets in determining the stress response. J. Pers. Soc. Psychol. 104, 716-733. doi: 10.1037/a0031201

De Berker, A. O., Rutledge, R. B., Mathys, C., Marshall, L., Cross, G. F., Dolan, R. J., et al. (2016). Computations of uncertainty mediate acute stress responses in humans. Nat. Commun. 7:10996. doi: 10.1038/ncomms10996

DeYoung, C. G. (2010). Personality neuroscience and the biology of traits. Soc. Person. Psychol. Compass 4, 1165-1180. doi: 10.1111/j.1751-9004.2010.00327.x

DeYoung, C. G. (2013). The neuromodulator of exploration: a unifying theory of the role of dopamine in personality. Front. Hum. Neurosci. 7:762. doi: 10.3389/fnhum.2013.00762

Drummond, S., and Brough, P. (2016). Proactive coping and preventive coping: evidence for two distinct constructs? Pers. Individ. Differ. 92, 123-127. doi: 10.1016/j.paid.2015.12.029

Ebstrup, J. F., Eplov, L. F., Pisinger, C., and Jørgensen, T. (2011). Association between the five factor personality traits and perceived stress: is the effect mediated by general self-efficacy? Anxiety Stress Coping 24, 407-419. doi: $10.1080 / 10615806.2010 .540012$

Epel, E. S., Blackburn, E. H., Lin, J., Dhabhar, F. S., Adler, N. E., Morrow, J. D., et al. (2004). Accelerated telomere shortening in response to life stress. Proc. Natl. Acad. Sci. U. S. A. 101, 17312-17315. doi: 10.1073/pnas.0407162101

Evans, A. M., and Krueger, J. I. (2009). The psychology (and economics) of trust. Soc. Personal. Psychol. Compass 3, 1003-1017. doi: 10.1111/j.1751-9004.2009.00232.x

Evans, A. M., and Revelle, W. (2008). Survey and behavioral measurements of interpersonal trust. J. Res. Pers. 42, 1585-1593. doi: 10.1016/j.jrp.2008.07.011

FeldmanHall, O., Raio, C. M., Kubota, J. T., Seiler, M. G., and Phelps, E. A. (2015). The effects of social context and acute stress on decision making under uncertainty. Psychol. Sci. 26, 1918-1926. doi: 10.1177/0956797615605807

Folkman, S., and Lazarus, R. S. (1985). If it changes it must be a process: study of emotion and coping during three stages of a college examination. J. Pers. Soc. Psychol. 48, 150-170. doi: 10.1037/0022-3514.48.1.150

Folkman, S., and Nathan, P. E. (2011). The Oxford handbook of stress, health and coping. New York: Oxford University Press. 
Freeston, M. H., Rhéaume, J., Letarte, H., Dugas, M. J., and Ladouceur, R. (1994). Why do people worry? Pers. Individ. Differ. 17, 791-802. doi: 10.1016/0191-8869(94)90048-5

Gentes, E. L., and Ruscio, A. M. (2011). A meta-analysis of the relation of intolerance of uncertainty to symptoms of generalized anxiety disorder, major depressive disorder, and obsessive-compulsive disorder. Clin. Psychol. Rev. 31, 923-933. doi: 10.1016/j.cpr.2011.05.001

Giordano, G. N., and Lindström, M. (2016). Trust and health: testing the reverse causality hypothesis. J. Epidemiol. Community Health 70, 10-16. doi: 10.1136/jech-2015-205822

Giordano, G. N., Mewes, J., and Miething, A. (2019). Trust and all-cause mortality: a multilevel study of US general social survey data (1978-2010). J. Epidemiol. Community Health 73, 50-55. doi: 10.1136/jech-2018-211250

Glanville, J. L., and Paxton, P. (2007). How do we learn to trust? A confirmatory tetrad analysis of the sources of generalized trust. Soc. Psychol. Q. 70, 230-242. doi: 10.1177/019027250707000303

Greenglass, E. R., and Fiksenbaum, L. (2009). Proactive coping, positive affect, and well-being: testing for mediation using path analysis. Eur. Psychol. 14, 29-39. doi: 10.1027/1016-9040.14.1.29

Greenglass, E. R., Schwarzer, R., Jakubiec, D., Fiksenbaum, L., and Taubert, S. (1999). "The proactive coping inventory (PCI): a multidimensional research instrument" in 20th International Conference of the Stress and Anxiety Research Society; July 12-14, 1999; Poland: Cracow, 1-18.

Guiso, L., Sapienza, P., and Zingales, L. (2004). The role of social capital in financial development. Am. Econ. Rev. 94, 526-556. doi: 10.1257/000282 8041464498

Hair, J. F. Jr., Black, W. C., Babin, B. J., and Anderson, R. E. (2014). Multivariate data analysis. 7th Edn. Essex: Pearson Education Limited.

Hale, W., Richmond, M., Bennett, J., Berzins, T., Fields, A., Weber, D., et al. (2016). Resolving uncertainty about the intolerance of uncertainty scale-12: application of modern psychometric strategies. J. Pers. Assess. 98, 200-208. doi: 10.1080/00223891.2015.1070355

Han, S. (2019). Social capital and perceived stress: the role of social context. J. Affect. Disord. 250, 186-192. doi: 10.1016/j.jad.2019.03.034

Hiraishi, K., Yamagata, S., Shikishima, C., and Ando, J. (2008). Maintenance of genetic variation in personality through control of mental mechanisms: a test of trust, extraversion, and agreeableness. Evol. Hum. Behav. 29, 79-85. doi: 10.1016/j.evolhumbehav.2007.07.004

Hooghe, M., Marien, S., and de Vroome, T. (2012). The cognitive basis of trust: the relation between education, cognitive ability and political trust. Intelligence 40, 604-613. doi: 10.1016/j.intell.2012.08.006

Kato, T. (2013). Frequently used coping scales: a meta-analysis. Stress. Health 31, 315-323. doi: 10.1002/smi.2557

Kawachi, I., Subramanian, S. V., and Kim, D. (2008). Social capital and health. New York: Springer Science.

King-Casas, B., Tomlin, D., Anen, C., Camerer, C. F., Quartz, S. R., and Montague, P. R. (2005). Getting to know you: reputation and trust in a two-person economic exchange. Science 308, 78-83. doi: 10.1126/science.1108062

Koranyi, N., and Rothermund, K. (2012). Automatic coping mechanisms in committed relationship: increased interpersonal trust as a response to stress. J. Exp. Soc. Psychol. 48, 180-185. doi: 10.1016/j.jesp.2011.06.009

Kotov, R., Gamez, W., Schmidt, F., and Watson, D. (2010). Linking "big" personality traits to anxiety, depressive, and substance use disorders: a metaanalysis. Psychol. Bull. 136, 768-821. doi: 10.1037/a0020327

Lazarus, R. S., and Folkman, S. (1984). Stress, appraisal, and coping. New York: Springer Publishing.

Lee, E. H. (2012). Review of the psychometric evidence of the perceived stress scale. Asian Nurs. Res. 6, 121-127. doi: 10.1016/j.anr.2012.08.004

Lee, K., and Ashton, M. (2004). Psychometric properties of the HEXACO personality inventory. Multivar. Behav. Res. 39, 329-358. doi: 10.1207/ s15327906mbr3902_8

Lee, K., and Ashton, M. C. (2005). Psychopathy, Machiavellianism, and narcissism in the five-factor model and the HEXACO model of personality structure. Pers. Individ. Differ. 38, 1571-1582. doi: 10.1016/j.paid.2004.09.016

Lee, K., and Ashton, M. C. (2014). The dark triad, the big five, and the HEXACO model. Pers. Individ. Differ. 67, 2-5. doi: 10.1016/j.paid.2014.01.048

Lupien, S. J., McEwen, B. S., Gunnar, M. R., and Heim, C. (2009). Effects of stress throughout the lifespan on the brain, behavior and cognition. Nat. Rev. Neurosci. 10, 434-445. doi: 10.1038/nrn2639
MacKinnon, D. P., Krull, J. L., and Lockwood, C. M. (2000). Equivalence of the mediation, confounding and suppression effect. Prev. Sci. 1, 173-181. doi: 10.1023/A:1026595011371

McEvoy, P., and Mahoney, A. (2012). To be sure, to be sure: intolerance of uncertainty mediates symptoms of various anxiety disorders and depression. Behav. Ther. 43, 533-545. doi: 10.1016/j.beth.2011.02.007

McQuaid, R. J., McInnis, O. A., Paric, A., Al-Yawer, F., Matheson, K., and Anisman, H. (2016). Relations between plasma oxytocin and cortisol: the stress buffering role of social support. Neurobiol. Stress. 3, 52-60. doi: 10.1016/j.ynstr.2016.01.001

Pechtel, P., and Pizzagalli, D. A. (2011). Effects of early life stress on cognitive and affective function: an integrated review of human literature. Psychopharmacol. 214, 55-70. doi: 10.1007/s00213-010-2009-2

Penley, J. A., and Tomaka, J. (2002). Associations among the big five, emotional responses, and coping with acute stress. Pers. Individ. Differ. 32, 1215-1228. doi: 10.1016/S0191-8869(01)00087-3

Raposa, E. B., Laws, H. B., and Ansell, E. B. (2015). Prosocial behavior mitigates the negative effects of stress in everyday life. Clin. Psychol. Sci. 4, 691-698. doi: $10.1177 / 2167702615611073$

Redmond, N., Richman, J., Gamboa, C. M., Albert, M. A., Sims, M., Durant, R. W., et al. (2013). Perceived stress is associated with incident coronary heart disease and all-cause mortality in low- but not high-income participants in the reasons for geographic and racial differences in stroke study. J. Am. Heart Assoc. 2:e00447. doi: 10.1161/JAHA.113.000447

Roesch, S. C., Aldridge, A. A., Huff, T. L. P., Langner, K., Villodas, F., and Bradshaw, K. (2009). On the dimensionality of the proactive coping inventory: 7, 5, 3 factors? Anxiety Stress Coping 22, 327-339. doi: 10.1080/106158 00802082304

Rotter, J. B. (1971). Generalized expectancies for interpersonal trust. Am. Psychol. 26, 443-452. doi: 10.1037/h0031464

Salim, S. (2014). Oxidative stress and psychological disorders. Curr. Neuropharmacol. 12, 140-147. doi: 10.2174/1570159x11666131120230309

Schneiderman, N., Ironson, G., and Siegel, S. D. (2005). Stress and health: psychological, behavioral, and biological determinants. Annu. Rev. Clin. Psychol. 1, 607-628. doi: 10.1146/annurev.clinpsy.1.102803.144141

Schwarzer, R., and Taubert, S. (2002). "Tenacious goal pursuits and striving toward personal growth: proactive coping" in Beyond coping: Meeting goals, visions, and challenges. ed. E. Frydenberg (New York: Oxford University Press), 19-35.

Sexton, K. A., Norton, P. J., Walker, J. R., and Norton, G. R. (2003). Hierarchical model of generalized and specific vulnerabilities in anxiety. Cogn. Behav. Ther. 32, 82-94. doi: 10.1080/16506070302321

Shiell, A., Hawe, P., and Kavanagh, S. (2020). Evidence suggests a need to rethink social capital and social capital interventions. Soc. Sci. Med. 257:111930. doi: 10.1016/j.socscimed.2018.09.006

Soto, C. J., and John, O. P. (2017). The next big five inventory (BFI-2): developing and assessing a hierarchical model with 15 facets to enhance bandwidth, fidelity, and predictive power. J. Pers. Soc. Psychol. 113, 117-143. doi: 10.1037/ pspp0000096

Straud, C., McNaughton-Cassill, M., and Fuhrman, R. (2015). The role of the five factor model of personality with proactive coping and preventative coping among college students. Pers. Individ. Differ. 83, 60-64. doi: 10.1016/j. paid.2015.03.055

Tabachnick, B. G., and Fidell, L. S. (2013). Using multivariate statistics. Boston: Pearson.

Takahashi, T., Ikeda, K., Ishikawa, M., Kitamura, N., Tsukasaki, T., Nakama, D., et al. (2005). Interpersonal trust and social stress-induced cortisol elevation. Neuroendocrinology 16, 197-199. doi: 10.1097/00001756-200502080-00027

Uslaner, E. M. (2002). The moral foundations of trust. New York: Cambridge University Press.

Van Lange, P. A. M. (2015). Generalized trust: four lessons from genetics and culture. Curr. Dir. Psychol. Sci. 24, 71-76. doi: 10.1177/0963721414552473

von Dawans, B., Fischbacher, U., Kirschbaum, C., Fehr, E., and Heinrichs, M. (2012). The social dimension of stress reactivity: acute stress increases prosocial behavior in humans. Psychol. Sci. 23, 651-660. doi: 10.1177/09567 97611431576

Walter, S. L., Seibert, S. E., Goering, D., and O’Boyle, E. H. (2019). A tale of two sample sources: do results from online panel data and conventional data converge? J. Bus. Psychol. 34, 425-452. doi: 10.1007/s10869-018-9552-y 
Wilson, A. E., and Darke, P. R. (2012). The optimistic trust effect: use of belief in a just world to cope with decision-generated threat. J. Consum. Res. 39, 615-628. doi: 10.1086/664499

Yamagishi, T., Akutsu, S., Cho, K., Inoue, Y., Li, Y., and Matsumoto, Y. (2015). Two-component model of general trust: predicting behavioral trust from attitudinal trust. Soc. Cogn. 33, 436-458. doi: 10.1521/soco. 2015.33.5.436

Yamagishi, T., and Yamagishi, M. (1994). Trust and commitment in the United States and Japan. Motiv. Emot. 18, 129-166. doi: 10.1007/ BF02249397

Yanagisawa, K., Masui, K., Furutani, K., Nomura, M., Ura, M., and Yoshida, H. (2011). Does higher general trust serve as a psychosocial buffer against social pain? An NIRS study of social exclusion. Soc. Neurosci. 6, 190-197. doi: $10.1080 / 17470919.2010 .506139$
Zhao, K., Ferguson, E., and Smillie, L. D. (2016). Prosocial personality traits differentially predict egalitarianism, generosity, and reciprocity in economic games. Front. Psychol. 7:1137. doi: 10.3389/fpsyg.2016.01137

Conflict of Interest: The authors declare that the research was conducted in the absence of any commercial or financial relationships that could be construed as a potential conflict of interest.

Copyright (C) 2020 Carlander and Johansson. This is an open-access article distributed under the terms of the Creative Commons Attribution License (CC BY). The use, distribution or reproduction in other forums is permitted, provided the original author(s) and the copyright owner(s) are credited and that the original publication in this journal is cited, in accordance with accepted academic practice. No use, distribution or reproduction is permitted which does not comply with these terms. 